\title{
Observação in vitro da ação dos isolados fúngicos Duddingtonia flagrans, Monacrosporium thaumasium e Verticillium chlamydosporium sobre ovos de Ascaris lumbricoides (Lineu, 1758)
}

\section{In vitro observation of the action of isolates of the fungi Duddingtonia flagrans, Monacrosporium thaumasium and Verticillium chlamydosporium on the eggs of Ascaris lumbricoides (Linnaeus, 1758)}

\author{
Fábio R. Braga ${ }^{1}$, Jackson V. Araújo ${ }^{1,2}$, Artur K. Campos ${ }^{3}$, Rogério O. Carvalho ${ }^{1}$, \\ André R. Silva ${ }^{1}$, Alexandre O. Tavela ${ }^{1}$ e Alessandro S. Maciel ${ }^{1}$
}

\section{RESUMO}

Observou-se a ação in vitro dos fungos nematófagos Duddingtonia flagrans, Monacrosporium thaumasium $e$ Verticillium chlamydosporium sobre ovos de Ascaris lumbricoides. Após sete, dez e quatorze dias de interação, o fungo promissor a ser utilizado no controle biológico de Asaris lumbricoides foi o Verticillium chlamydosporium (26-30\%). Os outros fungos não foram satisfatórios.

Palavras-chaves: Ascaris lumbricoides. Fungos nematófagos. Duddingtonia flagrans. Monacrosporium thaumasium. Verticillium chlamydosporium.

\section{ABSTRACT}

The in vitro action of the nematophagous fungi Duddingtonia flagrans, Monacrosporium thaumasium and Verticillium chlamydosporium on eggs of Ascaris lumbricoides was observed. After 7, 10 and 14 days of interaction, the fungus showing most promise for use in biologically control over Ascaris lumbricoides was Verticillium chlamydosporium (26-30\%). The other fungi did not present satisfactory results.

Key words: Ascaris lumbricoides. Nematophagous fungi. Duddingtonia flagrans. Monacrosporium thaumasium. Verticillium chlamydosporium.

No Brasil e no mundo, um alto número de pessoas está infectado por parasitas intestinais e, vários são os fatores sugeridos como causas: Uma condição sócio-econômica baixa, 0 aumento populacional, às constantes migrações internas a falta de saneamento básico, além de outros ${ }^{9}$. Embora a espécie Ascaris lumbricoides seja bem conhecida e muito comum em seres humanos, ainda hoje representa um problema de caráter extremamente importante para a saúde pública e de uma forma geral o problema é maior em países em desenvolvimento como o Brasil. A representatividade clínica desse parasita afeta principalmente crianças, notando-se sérias complicações 2 .

0 principal fator envolvido na transmissão é 0 ambiente uma vez que, os ovos de Ascaris lumbricoides quando eliminados nas fezes pelo hospedeiro definitivo não conseguem exercer papel de infectividade, ou seja, não possuem capacidade de infecção que só acontecerá após algumas semanas principalmente em lugares úmidos, sombreados e com temperatura alta, podendo contaminar a água e os alimentos. As estratégias para o controle desse parasita têm demonstrado principalmente: atenção as áreas com grande densidade populacional por serem as áreas de risco $0^{13}$.

Por outro lado, o controle biológico de nematóides realizado com fungos nematófagos é uma alternativa promissora e com resultados satisfatórios ${ }^{8}$. A vantagem de se aliar o controle biológico realizado com fungos nematófagos com o controle químico é a abrangente atuação sobre as formas infectantes presentes nas fezes, bem como sobre os nematóides que estão parasitando 0 animal ${ }^{11}$. Esses fungos se comportam como

\footnotetext{
1. Departamento de Medicina Veterinária, Universidade Federal de Viçosa, Viçosa, MG. 2. Pesquisador do CNPq. 3. Faculdade de Ciências Biológicas e da Saúde, Viçosa, MG. Projeto financiado pela FAPEMIG e CNPq

Endereço para correspondência: Dr. Jackson Victor de Araújo. Dept ${ }^{\circ}$ de Veterinária/ Universidade Federal de Viçosa. Av. Ph Rolfes s/n, 36570-000 Viçosa, MG, Tel: $55313899-1464$ 
antagonistas naturais de nematóides, sendo capazes de promover a captura, a morte ou mesmo a sua destruiçã̃ ${ }^{10}$.

Nos trabalhos de Lysek $^{5}$ e Lysek \& Sterba ${ }^{7}$ o fungo Verticillium chlamydosporium demonstrou ação sobre ovos de Ascaris lumbricoides, provando sua eficácia como controlador biológico.

0 presente trabalho procurou avaliar em diferentes intervalos a ação in vitro de isolados brasileiros de fungos nematófagos do gênero Duddingtonia flagrans, Monacrosporium thaumasium e Verticillium chlamydosporium, sobre ovos de Ascaris lumbricoides.

Quatro isolados de fungos nematófagos, um isolado de Duddingtonia flagrans, um de Monacrosporium thaumasium e dois Verticilium chlamydosporium foram mantidos em tubos de ensaio a $4^{\circ} \mathrm{C}$ contendo corn-meal-ágar 2\% (CMA 2\%) e no escuro. Esses isolados foram obtidos através da técnica de espalhamento do solo descrito por Duddington ${ }^{3}$, modificado por Santos e cols ${ }^{12}$.

Discos de cultura de $4 \mathrm{~mm}$ de diâmetro foram extraídos de isolados fúngicos mantidos em tubos de ensaio contendo CMA $2 \%$ transferidos para placas de Petri de $9 \mathrm{~cm}$ de diâmetro contendo $20 \mathrm{ml}$ de batata-dextrose-ágar 2\% (BDA 2\%) mantidos a $25^{\circ} \mathrm{C}$, no escuro e durante 10 dias. Após o crescimento dos isolados novos discos de cultura de $4 \mathrm{~mm}$ de diâmetro foram transferidos para placas de Petri de $9 \mathrm{~cm}$ diâmetro contendo $20 \mathrm{ml}$ de ágar-água 2\% (AA 2\%) e durante 10 dias.

Trinta mil ovos de Ascaris lumbricoides foram analisados morfologicamente ao microscópio óptico (10x) e a seguir mil ovos foram vertidos sobre a superfície das placas contendo apenas o meio AA $2 \%$ com isolados fúngicos e sem fungo (controle), permanecendo por um período de 14 dias. Nos intervalos de sete, dez e quatorze dias, cerca de cem ovos foram retirados de cada isolado e do controle com a ajuda de espátula e colocados em lâminas de vidro com uma gota de azul de Amam, e avaliados percentualmente de acordo com os parâmetros estabelecidos por Lysek5: sem alteração; efeito tipo 1, efeito lítico sem prejuízo morfológico à casca do ovo, onde hifas são observadas aderidas à casca; tipo 2, efeito lítico com alteração morfológica da casca e embrião do ovo, sem penetração de hifas através da casca e tipo 3, efeito lítico com alteração morfológica do embrião e da casca, além de penetração de hifas e colonização interna do ovo.

A atividade dos isolados Duddingtonia flagrans (AC001), Monacrosporium thaumasium (NF34a) e Verticillium chlamydosporium (VC1 e VC4) sobre os ovos de Ascaris lumbricoides, segundo os parâmetros estabelecidos por Lysek ${ }^{5}$, é representada nas Tabelas 1, 2 e 3.

Segundo Lysek ${ }^{5}$, o fungo que comprovadamente apresenta a maior atividade ovicida é aquele que demonstra os maiores percentuais do tipo 3, sendo potencialmente o fungo mais eficaz, dessa forma pôde-se avaliar que os isolados AC001 e NF34 não possuem atividade ovicida. Já o fungo Verticillium chlamydosporium apresentou maior efeito ovicida (Figuras 1 e 2) em relação aos isolados de Duddingtonia flagrans e Manacrosporium thaumasium, que não apresentaram qualquer efeito do tipo 2 e 3. Entretanto, no decorrer dos intervalos
Tabela 1 - Percentual de atividade ovicida dos fungos nematófagos, Duddingtonia flagrans, Monacrosporium thaumasium $e$ Verticillium chlamydosporium e o grupo controle sem fungos, sobre ovos de Ascaris lumbricoides no intervalo de 7 dias.

\begin{tabular}{lcccc}
\hline \multirow{2}{*}{ Grupos } & \multicolumn{4}{c}{ Intervalo 7 dias } \\
\cline { 2 - 5 } & sem alteração & efeito tipo $1^{*}$ & efeito tipo $2^{* * *}$ & efeito tipo 3*** \\
\hline AC 001 & 70,0 & 30,0 & 0,0 & 0,0 \\
NF 34a & 62,0 & 48,0 & 0,0 & 0,0 \\
VC 1 & 27,0 & 65,0 & 18,0 & 20,0 \\
VC 4 & 22,0 & 44,0 & 16,0 & 18,0 \\
Controle & 0,0 & 0,0 & 0,0 & 0,0 \\
\hline
\end{tabular}

*Efeito lítico sem prejuízo morfológico à casca do ovo, onde as hifas são observadas aderidas à casca.

**Efeito lítico com alteração morfológico da casca do ovo e do embrião, sem penetração de hifas através da casca.

***kefeito lítico com alteração morfológica da casca e do embrião, além da penetração de hifas e colonização interna do ovo.

Tabela 2 - Percentual de atividade ovicida dos fungos nematófagos, Duddingtonia flagrans, Monacrosporium thaumasium $e$ Verticillium chlamydosporium e o grupo controle sem fungos, sobre ovos de Ascaris lumbricoides no intervalo de 10 dias.

\begin{tabular}{lcccc}
\hline Grupos & \multicolumn{4}{c}{ Intervalo 10 dias } \\
\cline { 2 - 5 } & sem alteração & efeito tipo $1^{*}$ & efeito tipo $2^{* * *}$ & efeito tipo $3^{\text {**** }}$ \\
\hline AC 001 & 48,0 & 52,0 & 0,0 & 0,0 \\
NF 34a & 58,0 & 42,0 & 0,0 & 0,0 \\
VC 1 & 24,0 & 69,0 & 20,0 & 25,0 \\
VC 4 & 18,0 & 45,0 & 15,0 & 22,0 \\
Controle & 0,0 & 0,0 & 0,0 & 0,0 \\
\hline
\end{tabular}

*Efeito lítico sem prejuízo morfológico à casca do ovo, onde as hifas são observadas aderidas à casca.

**Efeito lítico com alteração morfológico da casca do ovo e do embrião, sem penetração de hifas através da casca.

***:Efeito lítico com alteração morfológica da casca e do embrião, além da penetração de hifas e colonização interna do ovo.

Tabela 3 - Percentual de atividade ovicida dos fungos nematófagos, Duddingtonia flagrans, Monacrosporium thaumasium e Verticillium chlamydosporium e o grupo controle sem fungos, sobre ovos de Ascaris lumbricoides no intervalo de 14 dias.

\begin{tabular}{lcccc}
\hline \multirow{2}{*}{ Grupos } & \multicolumn{4}{c}{ Intervalo 14 dias } \\
\cline { 2 - 5 } & sem alteração & efeito tipo $1^{*}$ & efeito tipo $2^{\text {*** }}$ & efeito tipo $3^{* * *}$ \\
\hline AC 001 & 32,0 & 68,0 & 0,0 & 0,0 \\
NF 34a & 38,0 & 62,0 & 0,0 & 0,0 \\
VC 1 & 23,0 & 22,0 & 26,0 & 30,0 \\
VC 4 & 22,0 & 32,0 & 20,0 & 26,0 \\
Controle & 0,0 & 0,0 & 0,0 & 0,0 \\
\hline
\end{tabular}

*Efeito lítico sem prejuízo morfológico à casca do ovo, onde as hifas são observadas aderidas à casca.

**Efeito lítico com alteração morfológico da casca do ovo e do embrião, sem penetração de hifas através da casca.

****kefeito lítico com alteração morfológica da casca e do embrião, além da penetração de hifas e colonização interna do ovo. 


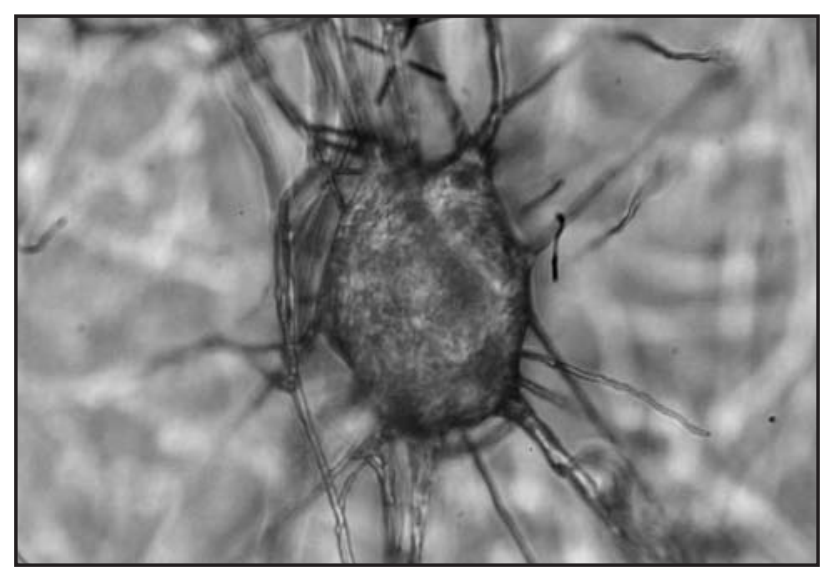

Figura 1 - Interação do fungo Verticillium chlamydosporium aos 21 dias com ovo de Ascaris lumbricoides (foto MO c/ aumento de 40X.).

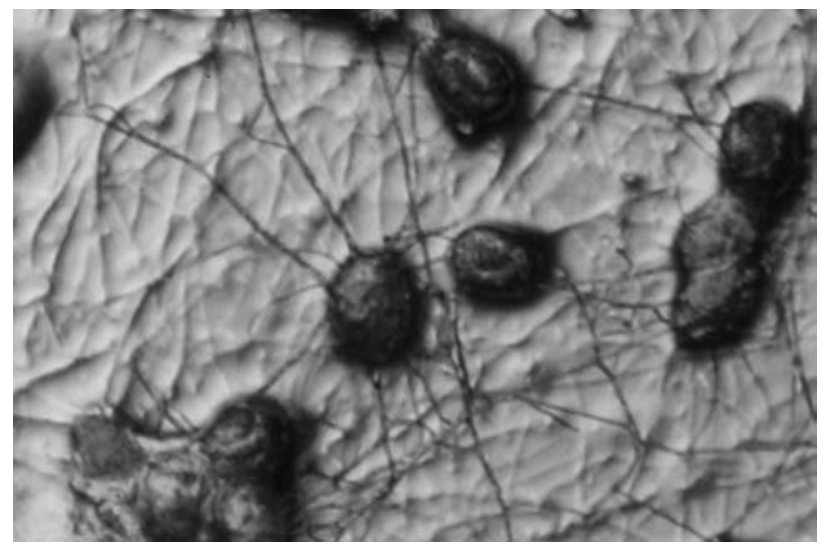

Figura 2 - Interação do fungo Verticillium chlamydosporium aos 14 dias com ovos de Ascaris lumbricoides (foto MO c/ aumento10X).

a ação ovicida dos isolados fúngicos (VC1 e VC4), aumentou de forma crescente, e comparando se com as observações de Lysek comprovase a eficácia do Verticillium chlamydosporium. Em relação à comprovação da eficácia dessefungo, como ovicida, pode-se mencionar os trabalhos desenvolvidos por Lysek ${ }^{5}$, Lysek e Sterba ${ }^{7}$ que, não apenas demonstraram atividade ovicida, mas também se equipararam ao trabalho realizado por de Freire e cols ${ }^{4}$ que demonstrou ação do Verticillium chlamydosporium sobre ovos, fêmeas e formas juvenis de Meloydogine incognita, um fitonematóide.

No trabalho desenvolvido por Araújo e cols ${ }^{1}$, com o isolado fúngico Paecilomyces lillacinus, o resultado encontrado de atividade ovicida do tipo 3, durante o intervalo de tempo de 7 dias, foi semelhante ao demonstrado no presente.

Foi observado que a atividade ovicida dos isolados fúngicos de Duddingtonia flagrans e Manacrosporium thaumasium, para os valores percentuais do tipo 1 e 2, equiparou-se também aos percentuais encontrados para os isolados de Arthrobotrys robusta e Arthrobotrys conoides, utilizados por Araújo e cols ${ }^{1}$ na ação sobre ovos embrionados de Toxocara canis.

0 presente trabalho é o primeiro relato da ação comparativa de fungos nematófagos das espécies Duddingtonia flagrans, Manacrosporium thaumasium e Verticillium chlamydosporium sobre ovos de Ascaris lumbricoides.

A importância desse estudo é sustentada pelo fato de que a transmissão de Ascaris lumbricoides ocorre principalmente através de ambiente contaminado ${ }^{13}$ e os fungos poderiam ser utilizados como ferramenta para a descontaminação ambiental por ovos de nematóide. Segundo os resultados apresentados, o fungo Verticillium chlamydosporium (isolados VC1 e vC4) demonstrou atividade ovicida sobre os ovos de Ascaris lumbricoides, sendo um fungo promissor a ser empregado no controle biológico desse nematóide.

\section{REFERÊNCIAS}

1. Araújo JV, Santos MA, Ferraz S. Efeito ovicida de fungos nematófagos sobre ovos embrionados de Toxocara canis. Arquivo Brasileiro de Medicina Veterinária e Zootecnia 47: 37-42, 1995.

2. Costa-Macedo LM, Costa MC, Almeida LM. Parasitismo pelo Ascaris lumbricoides em crianças menores de dois anos em comunidade aberta do Rio de Janeiro. Caderno de Saúde Publica 15:173-178, 1999.

3. Duddington CL. Notes on the thecnique of handling predaceous fungi. Transactions of British Mycology Society 38: 97-103, 1955.

4. Freire FCO, Bridge J. Parasitism of eggs, females and juveniles of Meloidogyne incognita by Paecilomyces lillacinus and Verticillium chlamydosporium. Embrapa, CAPTU. Phytopathology Brazilian 10:577-596, 1985.

5. Lysek $\mathrm{H}$. Classification of ovicide fungi according to type of ovicidity. Acta University Palack Olumue 76:9-13, 1976.

6. Lysek H. A scanning electron microscope study of the effects of ovicidal fungus on the eggs of Ascaris lumbricoides. Parasitology 77:139-141, 1978.

7. Lysek H, Sterba J. Colonization of Ascaris lumbricoides eggs by the fungus Verticillium chlamydosporium Goddard. Folia. Parasitology 38:255-259, 1991.

8. Mankau R. Biological control of nematode pest by natural enemies. Annual Review Phytopathology 18:415-440, 1980.

9. Morroni CA. Parasitoses Intestinais. In: Maakaroun MF, Souza RP, Cruz AR (eds) Manual de Adolescência. Diretoria de Publicações do SBP, Rio de Janeiro, p.670-695, 1989 .

10. Nordring-Hertz B, Jansson HB. Fungal development, predacity, and recognition of prey in nematode-destroying fungi. In: Klug MJ, Reddy CA (eds) Current prepectives in microbial ecology. Annual Society Microbiology, Wahshington, p. 327-333, 1984.

11. Ribeiro RR. Atividade predatória sobre larvas de Trichostrongílideos de isolados fúngicos do gênero Monacrosporium após a passagem pelo trato gastrointestinal de bovinos. Dissertação (Mestrado), Universidade Federal de Viçosa, Viçosa, MG, 2003.

12. Santos MA, Ferraz S, Muchove J. Detection and ecology of nematophagous fungi from Brazilian Soils. Nematologia Brasileira 15:121-134, 1991.

13. Tshikuka JG, Scott ME, Gray DK. Ascaris lumbricoides infection and environmental risk factories in urban African setting. Annual Tropical Medicine Parasitology 89:505-514, 1995. 